\title{
The Study of Dynamic Balancing for High-Speed Presses*
}

\author{
Cheng-Ho LI** and Pei-Lum TSO**
}

\begin{abstract}
In this paper, we proposed a concept of using both a linkage balancer and counterweight disks to reduce shaking force and shaking moment of high-speed mechanical presses. The linkage balancer is designed with analytic synthesis method for matching critical shaking forces at the right timing. Counterweight disks' positions and masses are designed with an optimum method that considers minimizing the magnitude and the fluctuation of the shaking effect. A practical design example on a Stephenson III six-bar linkage press is presented for illustration. The conclusion showed that the shaking force/moment could be effectively reduced. Comparatively, the linkage balancer and the counterweight disks are apt at dealing with shaking force and shaking moment, respectively.
\end{abstract}

Key Words: High-Speed Press, Dynamic Balancing, Vibration

\section{Introduction}

The press operating speed is increasing for providing more efficient production capability. At high operating speeds, ranged over $500 \sim 2000$ SPM (stroke per minutes), mechanical presses exhibit dynamic effects which may induce vibration and noise. For maintaining the reliable performance and high productivity of high-speed press, the dynamic effects must be seriously coped with; therefore, an applicable dynamic balancing strategy is necessary.

The unbalancing of press mechanisms is categorized in the reciprocating-type unbalancing since the ram keeps a periodically upward/downward motion. A conventional strategy for press balancing is to apply a linkage balancer mechanism. The linkage balancer is often a four-bar slider mechanism, and it is driven either by a crank or a rocker which is on the press mechanism, i.e., the linkage balancer and the press mechanism are both driven by the same input. The slider of the linkage balancer would go a reciprocating motion that results another shaking force to eliminate or reduce the original dynamic unbalance induced by the press mechanism. Based on our previous study ${ }^{(1)}$, this kind of slider-crank linkage balancer could effectively reduce the critical shaking forces down to 5\%, and the result said that we could apply slider-crank linkage balancer on different presses, but we must synthesize the dimensions

\footnotetext{
* Received 25th January, 2006 (No. 05-5145)

** Department of Power Mechanical Engineering, National Tsing Hua University, 101 Section 2 Kuang Fu Road, Hsinchu, Taiwan 300, Republic of China.

E-mail: d917704@oz.nthu.edu.tw;pltso@pme.nthu.edu.tw
}

and mass of such linkage balancer properly. Otherwise, the linkage balancer would not perform expectedly, but it might even worsen the dynamic problem of the press.

Besides adopting a linkage balancer, some other balancing strategies for machinery have been studied in the past. Arakelian ${ }^{(2)}$ presented an idea for complete balancing of linkages. He utilized a set of gear-driven inertia to achieve balancing, nevertheless, it seemed too complicated and costing to be introduced into mechanical presses. Yan ${ }^{(3)}$ proposed a four-bar linkage balancing optimal design by varying input crank rotating speed, but the approach is impracticable here as we know that most of regular mechanical presses are supposed to be driven by a constant rotary input (with a flywheel). Adding counterweights is another conventional way for dynamic balancing. $\mathrm{Chiou}^{(4)}$ proposed an optimum balancing design of mechanical presses for precision cutting by using counterweight disks, and the result showed that the shaking moment could be reduced to around 50\% (compared with the dynamic behavior before balancing).

Briefly summarizing, utilizing the linkage balancer and adopting the counterweight disk method are apt at dealing with shaking force and shaking moment of mechanical presses respectively. Few papers discussed the balancing performance with applying multiple methods at the same time. In this paper, we studied the dynamic balancing approach for high-speed presses; a reciprocating balancer and the counterweight disks are both applied simultaneously. First we designed the linkage balancer with analytic dimensional method; the main purpose is to match the critical shaking forces induced by the balancer and the press mechanism. Then we designed 


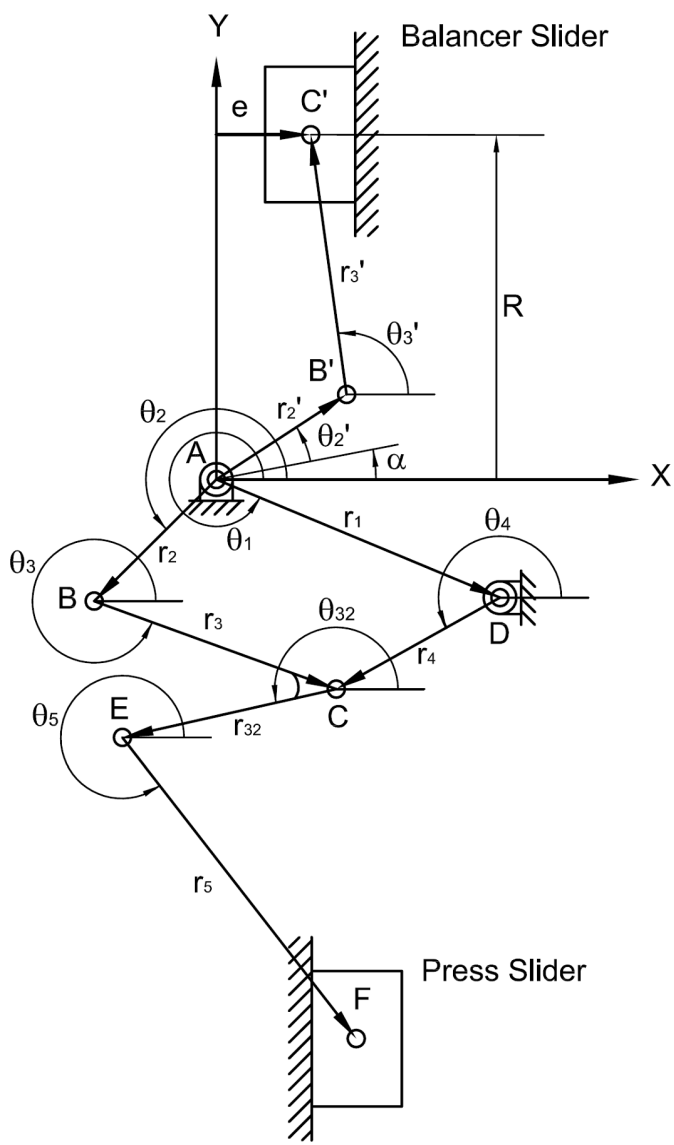

(a) Mechanism dimensions

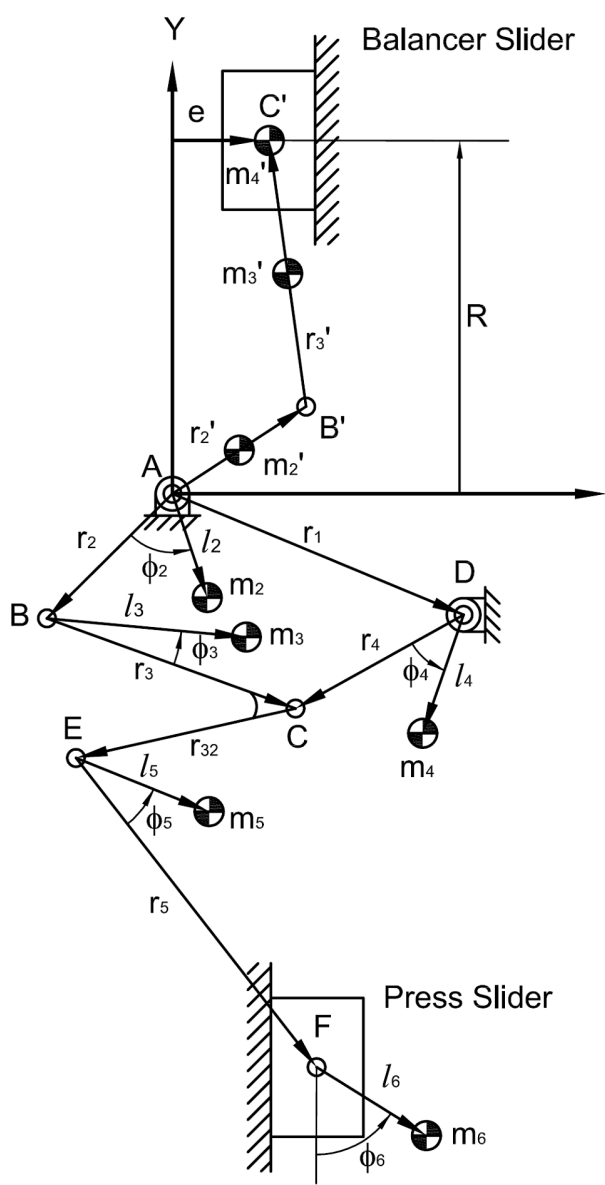

(b) Counterweights distribution

Fig. 1 Press mechanism and linkage balancer

counterweight disks' positions and masses with an optimum method. The optimum objective is to minimize both the magnitude and the fluctuation of the shaking effect. A design example on Stephenson III six-bar mechanical press is presented for illustration. The proposed method is applied and the balancing effects are investigated. The results show that using the linkage balancer and counterweight disks is advantageous to deal with both shaking force and shaking moment. Moreover, the proposed method would be practicable for any type of mechanical presses.

\section{Basic Arrangement}

Fig. 1 (a) shows the scheme of a press mechanism and a linkage balancer. The press mechanism consists of a Stephenson six-bar linkage, including a frame $r_{1}$ (AD) and links $r_{2}(\mathrm{AB}), r_{3}(\mathrm{BC}), r_{32}(\mathrm{CE}), r_{4}(\mathrm{DC}), r_{5}(\mathrm{EF})$ and a press slider (connected to the joint $\mathrm{F}$ ). The symbols $r_{i}$ represent link lengths, and $\theta_{i}$ indicates the angular orientation of the link measured counterclockwise from the positive $X$-axis. The linkage balancer consists of a slidercrank mechanism, including links $r_{2}^{\prime}\left(\mathrm{AB}^{\prime}\right), r_{3}^{\prime}\left(\mathrm{B}^{\prime} \mathrm{C}^{\prime}\right)$ and a balancer slider (connected to the joint $\mathrm{C}^{\prime}$ ). Similar to the press mechanism, $r_{i}^{\prime}$ and $\theta_{i}^{\prime}$ represents the length and an- gular orientation of each link for the linkage balancer. $\alpha$ is a reference angle where the $\theta_{2}^{\prime}$ being measured from. The link $r_{2}$ and $r_{2}^{\prime}$ are input links of the press mechanism and the linkage balancer respectively.

In this study, all links are assumed rigid and homogeneous, and the joint clearances and frictions are neglected for simplifying the mathematical model. The mass centers distribution of each link is shown in Fig. 2 (b). Referring to Fig. 2 (b), for the link $r_{i}$ of the press mechanism, $m_{i}$ represents the link mass, and $\phi_{i}$ and $l_{i}$ are the relative angular and linear positions of the mass center. For the link $r_{i}^{\prime}$ of the linkage balancer, $m_{i}^{\prime}$ represents the link mass which is assumed to be at the midpoint of each link.

Because the input links $r_{2}$ and $r_{2}^{\prime}$ are driven with the same rotary motion, the press slider and the balancer slider would go reciprocating motions in opposite directions simultaneously. Therefore, the linkage balancer is able to provide a balancing force for counteracting the shaking force induced by the press mechanism. To clarify different meanings of the inertia effects, the inertia effects induced by the press mechanism are regarded as "shaking force/moment", and those induced by linkage balancer or counterweight disks are regarded as "balancing 


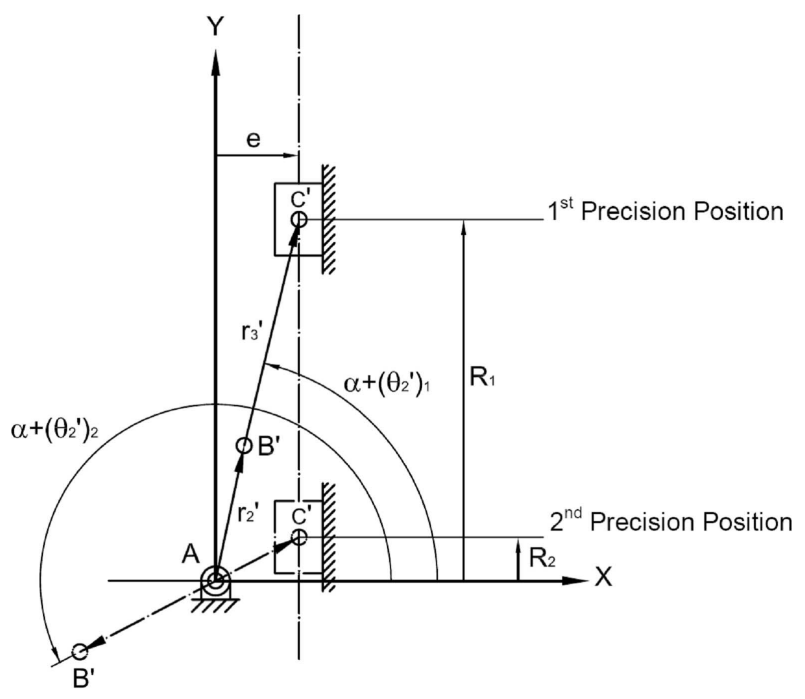

Fig. 2 Synthesis precision positions of linkage balancer

force/moment”.

\section{Linkage Balancer Design}

The main function of the linkage balancer is to reduce the shaking force. As we are using the slider-crank mechanism as the linkage balancer, the critical balancing forces would occur at its extreme positions ${ }^{(1)}$. Here we intend to use the slider-crank linkage balancer to counteract two critical values of the shaking force induced by the press mechanism. Before designing the linkage balancer, it is essential to obtain the inherent dynamic behaviors such as shaking force and moment. First, we need to know the timing of when critical shaking forces occur of the press mechanism, then we could utilize two-position function generation $^{(5)}$ synthesis with toggle constraints for determining the dimensions of the linkage balancer mechanism.

\subsection{Dimensional synthesis}

Referring to Fig. 2, the configurations of two precision points of the linkage balancer are shown. The two precision points are exactly arranged at extreme positions of the mechanism. At the $1^{\text {st }}$ precision position, the input link $r_{2}^{\prime}$ and the coupler link $r_{3}^{\prime}$ are both with the orientation angle of $\alpha+\left(\theta_{2}^{\prime}\right)_{1}$; meanwhile the slider reaches its maximum distance of $R_{1}$ measured from the $X$-axis. At the $2^{\text {nd }}$ precision position, the input link $r_{2}^{\prime}$ is with the orientation angle of $\alpha+\left(\theta_{2}^{\prime}\right)_{2}$ and overlapped with the link $r_{3}^{\prime}$; the slider reaches its minimum distance of $R_{1}$ measured from the $X$-axis. The vectors of links $r_{2}^{\prime}, r_{3}^{\prime}, e$ and $R$ form a vector loop, and two scalar equalities of components in $X$ and $Y$ directions could be obtained. After eliminating the variable $\theta_{3}^{\prime}$, the loop equation then could be written as Eq. (1). By investigating the geometric relations of the precision positions in Fig. 2, the toggle constraint of the linkage balancer mechanism could be written as Eq. (2).

$$
\left(r_{3}^{\prime}\right)^{2}=\left(r_{2}^{\prime}\right)^{2}+\left(R_{i}\right)^{2}+e^{2}-2 R_{i}\left(r_{2}^{\prime}\right) \cos \left[\alpha+\left(\theta_{2}^{\prime}\right)_{i}\right]
$$

$$
\begin{gathered}
-2 e\left(r_{2}^{\prime}\right) \sin \left[\alpha+\left(\theta_{2}^{\prime}\right)_{i}\right] \\
R_{i} \cdot \tan \left[\alpha+\left(\theta_{2}^{\prime}\right)_{i}\right]=e
\end{gathered}
$$

In Eq. (1), $r_{2}^{\prime}, r_{3}^{\prime}, e$ and $\alpha$ are variables to be solved. The symbols $r_{2}^{\prime}$ and $r_{3}^{\prime}$ represent link lengths as we mentioned before, and the unknown variable " $e$ " represents the slider offset (measured from $Y$-axis). The variable $\alpha$ is a reference angle. Mathematically, $\alpha$ is a trivial variable to fulfill the equation numbers, but actually it could be regarded as the orientation relation of link $r_{2}$ and $r_{2}^{\prime(1)}$.

Substituting $i=1$ and 2 (representing 1 st and 2 nd precision points respectively) into Eqs. (1) and (2), the equations would yield dimensions of the linkage balancer. Meanwhile, we should notice that the stroke of the linkage balancer could be specified with assigning slider positions, $R_{1}$ and $R_{2}$. This is advantageous for designers to take account of the space requirement of the linkage balancer. The said design conditions of two crank angles $\left(\theta_{2}^{\prime}\right)$ with two corresponding slider positions are regarded as the inputs for the synthesis. The solved dimensions include the crank length, $r_{2}^{\prime}$, the coupler length, $r_{3}^{\prime}$, the offset, $e$, and the reference angle, $\alpha$.

\subsection{Balancing force}

After dimensional synthesis design, the slider of the linkage balancer would reach its extreme positions exactly at the timing while two critical shaking forces occur. However, it is essential to consider the balancing force which the linkage balancer would actually produce. As shown in Fig. 1 (b), supposing that the mass center of the link $r_{3}^{\prime}$ is located at the midpoint of $\mathrm{B}^{\prime} \mathrm{C}^{\prime}$, according to the equivalent inertia concept, we could divide the mass $m_{3}^{\prime}$ equally into two point masses $\left(m_{B}^{\prime}\right.$ and $\left.m_{C}^{\prime}\right)$ at $\mathrm{B}^{\prime}$ and $\mathrm{C}^{\prime}$. The balancing force model in $Y$-axis direction could be derived with fundamental dynamic analysis straightforwardly, as written in Eq. (3):

$$
\begin{aligned}
F_{y} & =m_{2}^{\prime} \frac{r_{2}^{\prime}}{2} \omega^{2} \cos \theta+m_{B}^{\prime} r_{2}^{\prime} \omega^{2} \cos \theta-\left(m_{C}^{\prime}+m_{4}^{\prime}\right) \\
& \times\left(-r_{2}^{\prime} \omega^{2} \cos \theta-\frac{\left(r_{2}^{\prime}\right)^{2}}{r_{3}^{\prime}} \omega^{2} \cos 2 \theta-\frac{r_{2}^{\prime}}{r_{3}^{\prime}} \omega^{2} \cdot e \cdot \sin \theta\right)
\end{aligned}
$$

Where $\theta=\alpha+\theta_{2}^{\prime}, m_{B}^{\prime}=m_{C}^{\prime}=0.5 m_{3}^{\prime}$.

In the Eq. (3), the symbol $\theta$ is the angle of the link $r_{2}^{\prime}$, and $\omega$ is the angular speed of the link $r_{2}^{\prime}$. The parameters $m_{B}^{\prime}$ and $m_{C}^{\prime}$ are equivalent masses distributed at the ends of the coupler link $\mathrm{A}^{\prime} \mathrm{B}^{\prime}$, and $m_{4}^{\prime}$ is the mass of the balancer slider. The Eq. (3) helps one to decide proper masses of the links and the slider of the linkage balancer.

\section{Optimal Design of Counterweight Disks}

Adding counterweight disks on the press mechanism is a way to change the intrinsic mass distribution but not affect the strength of the linkage system. By doing so, we can say that the dynamic behavior of the press mechanism 
is re-arranged. The design of counterweight disks should be considered prudently; here we introduce an optimization design for obtaining the most superior result.

Defining $I_{i}$ as the moment inertia of each link $r_{i}$ on the press mechanism, the shaking force and shaking moment of the concerned press mechanism could be expressed as following equations.

$$
\begin{aligned}
& F=-\sum_{i=2}^{7} m_{i} a_{g i} \\
& M_{R}=-\sum_{i=2}^{7}\left(R_{g i} \times m_{i} a_{g i}+I_{i} \alpha_{i}\right)
\end{aligned}
$$

Where $a_{g i}$ and $\alpha_{i}$ are the acceleration of the mass center and angular acceleration of link $i$. The shaking moment is the sum of the inertial torques and the torques of the inertial forces with respect to the reference point. $R_{i}$ is the position vector of the mass center of link $i$ with respect to the reference point $R\left(x_{r}, y_{r}\right)$.

Some other related functions are defined as well for the calculation afterward. Through a cycle of the press mechanism, $\bar{F}$ is the root-mean-squared shaking force, and $\bar{M}_{R}$ is the root-mean-squared shaking moment with respect to the reference point $R . \bar{F}$ and $\bar{M}_{R}$ are for evaluating the average magnitudes of the shaking effects. Furthermore, $\Delta F$ and $\Delta M_{R}$ represent the ranges of shaking force and shaking moment, respectively, that measure the variations or fluctuations of the shaking effects.

$$
\begin{aligned}
& \bar{F}=\sqrt{\frac{1}{2 \pi} \int_{0}^{2 \pi} F^{2}\left(\theta_{2}\right) d \theta_{2}} \\
& \bar{M}_{R}=\sqrt{\frac{1}{2 \pi} \int_{0}^{2 \pi} M_{R}^{2}\left(\theta_{2}\right) d \theta_{2}} \\
& \Delta F=F_{\max }-F_{\min } \\
& \Delta M=M_{\max }-M_{\min }
\end{aligned}
$$

In this study, Hertrich's ${ }^{(6)}$ claim is adopted that if the counterweight disk on the link with a fixed pivot in such a way that the contour of the disk is tangent to the center of the fixed pivot, then the increase in moment of inertia can be minimized. Therefore, the counterweight disks are to be installed on the links with fixed pivot, the links $r_{2}$ (AB) and $r_{4}$ (DC) as shown in Fig. 1 (a). The configuration of the link with the counterweight disk is shown in Fig. 3. $m_{b i}$ and $l_{b i}$ indicate the mass and the radius of the disk, respectively. $\beta_{i}$ represents the angular position, and $l_{i}$ represents the distance of the mass center, both relative to a pivot. These arrangements can be also directly substituted into Eqs. (4) and (5) to calculate the shaking force and shaking moment of the system.

\section{1 Design variables}

The counterweight disks are supposed to be placed on link 2 and link 4. Four design variables are listed below:

$l_{b 2}:$ distance from the center of disk2 to the pivot A

$l_{b 4}:$ distance from the center of disk4 to the pivot D

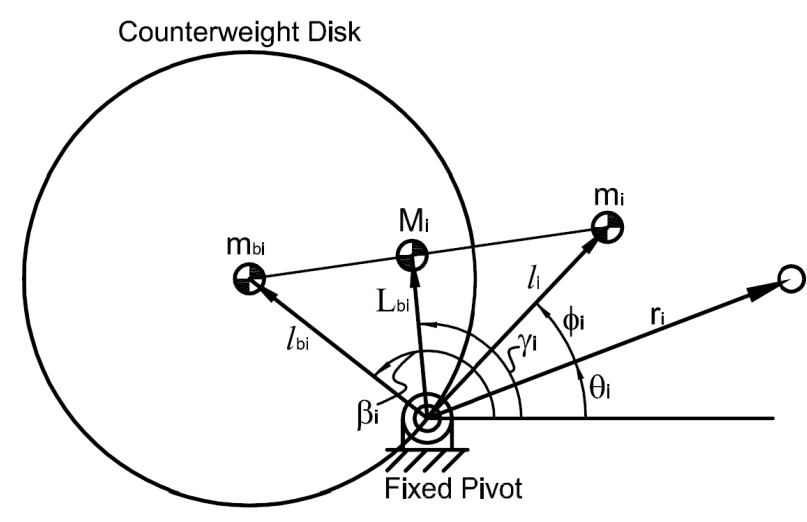

Fig. 3 Link with the counterweight disk

$\beta_{2}$ : relative angular position of disk2 with respect to link 2

$\beta_{4}$ : relative angular position of disk4 with respect to link 4

The radius of each disk is equal to their distance from the fixed pivots by reason of that each disk's contour is required to be tangent to the centers of the fixed pivots.

\subsection{Objective function}

To minimize the magnitudes and fluctuations of the shaking effects, we are considering the relative ratio of the shaking effects before and after being balanced with counterweight disks. The multi-objective function written as Eq. (10) is used. Explicitly speaking, the first and the third terms are concerning to the magnitudes, and the other two terms are concerning to the fluctuations of the shaking effects. The smaller value of the objective function $f_{o b j}$ indicates that the better balancing outcome is achieved.

$$
\begin{gathered}
f_{o b j}=w_{1} s_{1} \frac{(\bar{F})_{b}}{(\bar{F})_{u}}+w_{2} s_{2} \frac{(\Delta F)_{b}}{(\Delta F)_{u}}+w_{3} s_{3} \frac{\left(\bar{M}_{R}\right)_{b}}{\left(\bar{M}_{R}\right)_{u}} \\
+w_{4} s_{4} \frac{\left(\Delta M_{R}\right)_{b}}{\left(\Delta M_{R}\right)_{u}}
\end{gathered}
$$

In Eqs. (10), $w_{1} \sim w_{4}$ are weighting factors; $s_{1} \sim s_{4}$ are scaling factors. The subscripts " $b$ " and " $u$ " mean "balanced" and unbalanced", respectively. Thus, the objective function is actually an index of the balancing effect in terms of the shaking effect ratio, before and after balanced. Using the objective function, the optimal placements of two counterweight disks could be figured out.

\subsection{Constraints}

The constraints are the allowable ranges of the design variables. In this optimal design problem, the constraints are mainly dominated by the space limitation of the press. Hence, the constraints can be simply expressed as below:

$$
\begin{aligned}
& l_{\min } \leq l_{b i} \leq l_{\max } \\
& \beta_{\min } \leq \beta_{i} \leq \beta_{\max }
\end{aligned}
$$

The upper and lower limitations of $l_{b i}$ and $\beta_{i}$ depend on different presses space restrictions. 
Table 1 Dimension of the Stephenson six-bar press mechanism

\begin{tabular}{|c|c|c|c|c|c|c|}
\hline i & $\begin{array}{c}\mathbf{r}_{\mathbf{i}} \\
(\mathbf{m m})\end{array}$ & $\begin{array}{c}\mathbf{m}_{\mathbf{i}} \\
(\mathbf{k g})\end{array}$ & $\begin{array}{c}l_{\mathrm{i}} \\
(\mathbf{m m})\end{array}$ & $\begin{array}{c}\phi_{\mathrm{i}} \\
(\mathrm{deg})\end{array}$ & $\underset{\left(\mathbf{k g}-\mathrm{m}^{2}\right)}{\mathbf{I}_{\mathbf{i}}}$ & $\begin{array}{c}\theta_{\mathrm{i}} \\
(\mathrm{deg})\end{array}$ \\
\hline 1 & 427 & - & - & - & - & 139 \\
\hline 2 & 95 & 42 & 35.2 & 0 & 1.03 & - \\
\hline 3 & 297 & \multirow{2}{*}{89} & 115.3 & -28 & \multirow{2}{*}{0.65} & - \\
\hline 32 & 617 & & - & - & & - \\
\hline 4 & 273 & 62 & 140 & 0 & 0.37 & - \\
\hline 5 & 487 & 20 & 212.3 & 0 & 0.39 & - \\
\hline 6 & - & 140 & - & - & - & - \\
\hline
\end{tabular}

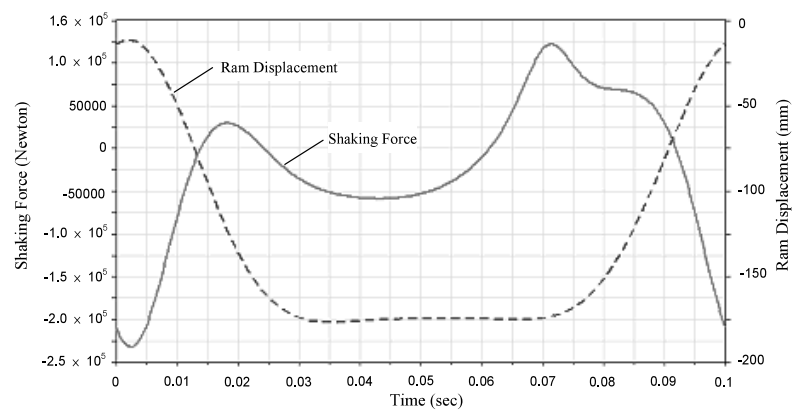

Fig. 4 Shaking force of the Stephenson six-bar press

\section{Design Example}

To illustrate the approach proposed, a design example is presented. The object is a Stephenson six-bar press. The skeleton is on Fig. 1 (a). The main purpose is to design a linkage balancer and figure out the optimal counterweight disks arrangement.

Table 1 shows the dimensions of a Stephenson III Sixbar press. The simulation of the press operation at 600 SPM has been accomplished by ADMAS CAE software, and the shaking force curve is as shown in Fig. 4. According to the dynamic analysis results, we know that the critical shaking forces occur while the crank angle reaches $\theta_{1}=199^{\circ}$ and $\theta_{2}=50^{\circ}$. These two angles are the basic design inputs of the dimensional synthesis for the linkage balancer. Considering the space limitation of the press, the extreme positions of the linkage balancer are decided as $R_{1}=1000(\mathrm{~mm})$ and $R_{2}=310(\mathrm{~mm})$. Substituting the inputs of $\theta_{1}, \theta_{2} R_{1}$ and $R_{2}$ into Eqs. (1) and (2), the dimensions of the linkage balancer could be solved. Afterward the linkage balancer is assured to reach its two extreme positions with corresponding to two critical values of the shaking force.

The maximum mass of the balancer slider is set equally as the mass of press slider, $500 \mathrm{~kg}$. After calculating with balancing force model, Eq. (3), the residual masses could be determined. All dimensions of the linkage balancer designed are listed is Table 2 .

The optimization result of the counterweight disks is shown in Table 3.

\section{Discussion}

We utilized the ADAMS CAE software to simulate
Table 2 Designed dimensions of the linkage balancer

\begin{tabular}{|c|c|c|c|}
\hline i & $\begin{array}{c}\mathbf{r}_{\mathbf{i}} \\
(\mathbf{m m})\end{array}$ & $\begin{array}{c}\mathbf{m}_{\mathbf{i}}^{\prime} \\
(\mathbf{k g})\end{array}$ & $\begin{array}{c}\mathbf{I}_{\mathbf{i}} \\
\left(\mathrm{kg}-\mathrm{m}^{2}\right)\end{array}$ \\
\hline $2^{\prime}$ & 223.4 & 42 & 1.03 \\
\hline 3 ' & 1012.7 & 40 & 3.41 \\
\hline $4 '$ & - & 35 & - \\
\hline \multicolumn{4}{|c|}{$\mathrm{e}=726.5(\mathrm{~mm})$} \\
\hline
\end{tabular}

Table 3 Optimization result of the counterweight disks

\begin{tabular}{c|c|c|c}
\hline $\begin{array}{c}l_{\mathrm{b} 2} \\
(\mathbf{m m})\end{array}$ & $\begin{array}{c}l_{\mathrm{b} 4} \\
(\mathbf{m m})\end{array}$ & $\begin{array}{c}\beta_{2} \\
(\mathbf{d e g})\end{array}$ & $\begin{array}{c}\beta_{4} \\
(\mathbf{d e g})\end{array}$ \\
\hline 63 & 87 & 33 & 42 \\
\hline
\end{tabular}

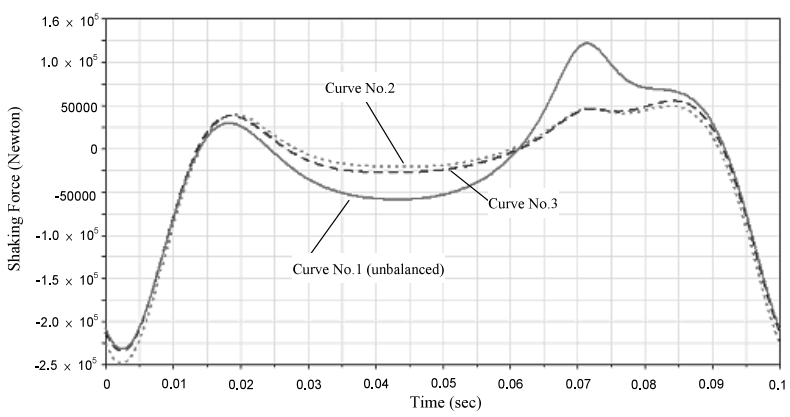

Fig. 5 Shaking force balancing result

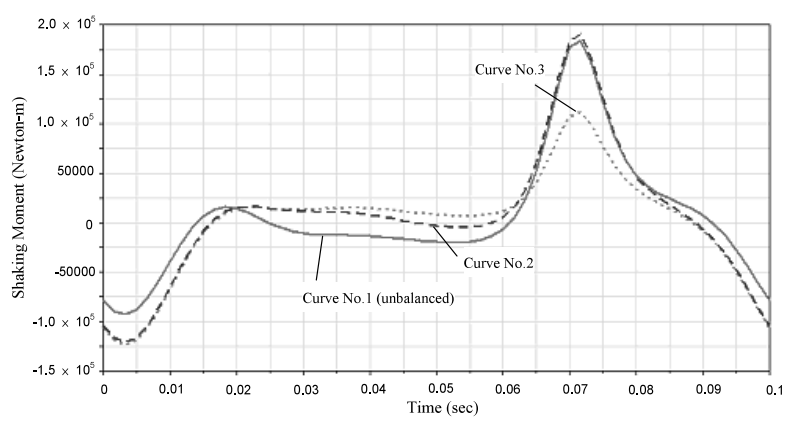

Fig. 6 Shaking moment balancing result

the dynamic behaviors of the press mechanism. The results of comparison in shaking force/moment are shown in Figs. 5 and 6. The operating speed of the press is 600 SPM.

In Fig. 5, the first solid curve (No.1) shows the shaking force before balanced. After installing a linkage balancer, the extreme values are effectively reduced and becomes as shown in the second curve (No.2). The third curve (No.3) represents the shaking force after both installing linkage balancer and adding the optimal counterweight disks. The result shows that the counterweight disks make little affection for suppressing the magnitude of shaking force, but they do alleviating the fluctuation of shaking force.

In Fig. 6, the first solid curve (No.1) shows the shaking moment before balanced. After installing a linkage balancer, the shaking moment becomes the curve (No.2); 
the shape of the shaking moment curve changed a little bit, not much improvement has been done by the linkage balancer. However, adding counterweight disks with optimal consideration, not only the magnitude but also the fluctuation of the shaking moment is suppressed effectively, as shown in the third curve (No.3). The result implies that the counterweight disks, moving in circular motion, are more apt at improving the shaking moment than shaking force.

\section{Conclusion}

The balancing strategy combining a linkage balancer and counterweight disks is studied in this paper. A design method with dimensional synthesis notion for the linkage balancer is proposed. An optimal counterweight disks arrangement is discussed. A design example is illustrated, and the balance effect is evaluated by simulation. The results are summarized as following points.

1. The linkage balancer could effectively reduce the range of two critical shaking forces down to less than $10 \%$ of the original.

2. The counterweight disks are proved to be capable of reducing the value and the fluctuation of the shaking moment.

3. Applying both the linkage balancer and the counterweight disks could counterbalance the dynamic effects of a press in aspects of shaking force and shaking moment concurrently.

4. The approach proposed in this paper can be utilized for designing dynamic balancing system for any kind of presses or other reciprocating mechanisms.

\section{Acknowledgement}

The authors are grateful to Chin Fong Industrial Company for their supporting.

\section{References}

( 1 ) Li, C.H., Tso, P.L. and Tsai, B., Linkage Balancer Mechanism Design for Mechanical Presses, Proc. of the 20th National Conference of CSME on Mech. Eng., Vol.C, No.C01-10 (2003), pp.75-81.

(2) Arakelian, V.H. and Smith, M.R., Complete Shaking Forces and Shaking Moment Balancing of Linkages, Mechanism and Machine Theory, Vol.34 (1999), pp.1141-1153.

( 3 ) Yan, H.S. and Soong, R.C., Kinematic and Dynamic Design of Four-Bar Linkage by Counterweighing with Variable Input Speed, Mechanism and Machine Theory, Vol.36 (2001), pp.1150-1071.

( 4 ) Chiou, S.T., Bai, G.J. and Chang, W.K., Optimum Balancing Design of the Drag-link Drive of Mechanical Presses for Precision Cutting, Int. J. of Machine Tools and Manufacture, Vol.38, No.3 (1998), pp.131-141.

( 5 ) Barker, C.R. and Tso, P.L., Characteristic Surface for Three Position Function Generation with Planar Four Bar Mechanism, Int. J. Mechanisms, Transmissions and Automation in Design, Trans. ASME, Vol.111 (1989), pp.104-109.

( 6 ) Hertrich, F.R., How to Balance High-Speed Linkages Using Minimum-Inertia Counterweights, Machine Design, Vol.35, No.6 (1963), pp.160-164. 